\title{
A novel detachable filter to prevent thromboembolism during endovascular surgery
}

\author{
Kyokun Uehara, MD, ${ }^{\mathrm{a}}$ Akira Marui, MD, PhD, ${ }^{\mathrm{a}}$ Yoshimasa Nagata, ME, ${ }^{\mathrm{b}}$ Mitsuru Sato, ME, ${ }^{\mathrm{b}}$ \\ Takahide Takeda, MD, PhD, ${ }^{\mathrm{a}}$ Tadashi Ikeda, MD, PhD, ${ }^{\mathrm{a}}$ Ryuzo Sakata, MD, PhD, ${ }^{\mathrm{a}}$ and Kanji Inoue, $\mathrm{MD}^{\mathrm{b}, \mathrm{c}}$
}

Objective: Thromboembolism remains a serious complication during endovascular surgery. Commercially available filter devices, which are unified with the stenting systems, provide short-time performance owing to the adhesion of thrombus to the filters themselves. We have, therefore, developed a new detachable filter that can be used in all major aortic branches and shows greater longevity. The present study assessed the efficacy of the new detachable filter and examined the feasibility of deploying and retrieving the filters.

Methods: We first performed in vitro studies. Our experimental flow model used silicon tubing to simulate the aortic branches. Polystyrene-divinylbenzene microspheres (100 and $200 \mu \mathrm{m}$ in diameter), which simulated embolic particles, were injected into the tubing after the detachable filter was deployed. The capture efficacy (number of microspheres trapped in the detachable filter/total injected microspheres) was calculated. In the in vivo studies, the detachable filters were implanted into the carotid, visceral, and renal arteries of 5 mongrel dogs. Angiography was performed every 30 minutes. At 5 hours after implantation, each detachable filter was retrieved by a gooseneck snare catheter.

Results: In the in vitro studies, our detachable filters showed high capture efficacy, capturing $99.2 \%$ of the $100-\mu \mathrm{m}$ microspheres and $99.4 \%$ of the $200-\mu \mathrm{m}$ microspheres. In the in vivo studies, all detachable filters were successfully deployed into the major branches. Each angiographic study revealed smooth flow without any embolic obstruction of the filter. At 5 hours after deployment, all devices were completely retrieved by the snare catheter without aortic injury.

Conclusions: The new detachable filter showed high efficacy in capturing the particles. All detachable filters were successfully deployed for 5 hours, and the filters were retrieved from the aortic branches without any complications. This novel detachable filter can help prevent serious distal thromboembolism during endovascular surgery. (J Thorac Cardiovasc Surg 2012;144:1399-403)

Endovascular surgery, such as aortic stent grafting or transcatheter aortic valve implantation, is emerging as an alternative treatment of thoracic aortic aneurysms and aortic stenosis in high-risk patients. ${ }^{1-5}$ However, distal embolic events are major complications of these procedures. ${ }^{5-8}$

Distal protection filters are routinely used to prevent embolic stroke in carotid artery stenting. ${ }^{9,10}$ Many studies have demonstrated the effectiveness of these protective filters. ${ }^{11-14}$ However, their use is limited to stenting operations, because they are combined with the stenting system. In addition, the longevity of the filters is poor because of thrombus formation on the filters themselves.

From the Department of Cardiovascular Surgery, ${ }^{a}$ Kyoto University Graduate School of Medicine, Kyoto, Japan; PTMC Institute, ${ }^{\mathrm{b}}$ Kyoto, Japan; and Department of Cardiovascular Surgery, ${ }^{\mathrm{c}}$ Shimabara Hospital, Kyoto, Japan.

Disclosures: Dr Kanji Inoue developed and created the detachable filter and holds all patents for it.

Received for publication July 7, 2011; revisions received Dec 2, 2011; accepted for publication Jan 4, 2012; available ahead of print Feb 3, 2012.

Address for reprints: Akira Marui, MD, PhD, Department of Cardiovascular Surgery,

Kyoto University Graduate School of Medicine, 54 Shogoin Kawahara, Sakyo,

Kyoto 606-8507 Japan (E-mail: marui@kuhp.kyoto-u.ac.jp).

$0022-5223 / \$ 36.00$

Copyright (C) 2012 by The American Association for Thoracic Surgery

doi:10.1016/j.jtcvs.2012.01.025
Therefore, we developed a detachable intra-aortic filter device with a new coating system to increase its durability. We designed the detachable filters such that after deployment in the targeted aortic branches, the filters could prevent distal thromboembolism during endovascular surgery without hindering the main operation.

In the present study, we assessed the efficacy of our new detachable filter and examined the feasibility of deploying and retrieving the filters using the canine cerebral, visceral, and renal arteries.

\section{METHODS}

\section{New Detachable Filter}

Our novel device consists of a nickel-titanium frame with a polyester mesh (Figure 1). The filter itself is composed of a polyester mesh with a new antithrombogenic coating system that inhibits both the enzymatic activity of thrombin and platelet adhesion (Toray Industries, Tokyo, Japan). Each pore in the polyester mesh is $100 \mu \mathrm{m}$ in diameter. The polyester yarn is $27 \mu \mathrm{m}$ in diameter. The mesh filter is mounted on a self-expandable nickel-titanium frame to allow the filter to conform to the interior diameter of each branch artery. The diameter of the frame ring is $8 \mathrm{~mm}$, which is designed to be $20 \%$ larger than the diameter of the canine aortic branches at the landing zone of the filter device. The frame ring is connected to a stainless steel shaft $(0.15 \mathrm{~mm}$ in diameter $)$ with 4 polyester threads. A slidable polyimide sleeve is set over the stainless steel shaft, together 
with the 4 polyester threads. The shaft is $30 \mathrm{~mm}$ in length, and its proximal end is bent into a hook shape. A J-shaped spring coil is attached to the distal tip of the shaft. The filter is preloaded in the delivery sheath, which is composed of a $4.5 \mathrm{~F}$ polytetrafluoroethylene sheath as attached to a $3 \mathrm{~F}$ shaft. To push out the filter from the delivery sheath, a 0.014-in. stainless steel wire with a plate on its tip, designated the "pushing wire," is put in advance in the delivery sheath at a position just proximal to the hooked wire of the preloaded filter.

\section{Deployment and Retrieval of Detachable Filter Device}

To deploy the filter, a $6 \mathrm{~F}$ sheath catheter (Johnson \& Johnson, New Brunswick, NJ) was inserted, and a $6 \mathrm{~F}$ guiding catheter (Johnson \& Johnson) was passed through the sheath catheter up to the thoracic aorta until its distal end reached the targeted branch of the aorta. Next, the filter system (the delivery sheath storing the preloaded filter and the pushing wire) was introduced into the guiding catheter. Once the J-shaped spring coil tip reached the end of the guiding catheter, its direction was adjusted by wrenching the shaft of the delivery sheath such that it can help to guide the filter system into the targeted artery. After the landing zone for the filter was secured, the filter was released and opened successively by pulling back the delivery sheath while the pushing wire was kept in place. The $6 \mathrm{~F}$ guiding catheter, in which the delivery sheath and pushing wire had been stored, was then removed from the $6 \mathrm{~F}$ sheath catheter.

To retrieve the filter device smoothly, an Amplatz gooseneck snare (Microvena, White Bear Lake, Mich) was passed through a $4 \mathrm{~F}$ catheter (Figure 2) to grip the hooked wire at the end of the shaft of the filter. The $4 \mathrm{~F}$ catheter was then pushed up until it abutted the polyimide slidable sleeve. The sleeve was then moved distally to gather the 4 polyester threads along the shaft whereby the ring frame and the mesh of the filter were folded inward. The completely folded filter was then withdrawn through the $6 \mathrm{~F}$ sheath catheter.

\section{In Vitro Studies}

The experimental flow model consisted of combined silicone tubing in 2 sizes: 1 with a 10-mm inner diameter to simulate the thoracic aorta and 1 with a 6-mm inner diameter to simulate an aortic branch such as the carotid artery, renal artery, or visceral artery (Figure 3). A roller pump (Capiox, SP-101; Terumo, Tokyo, Japan) was used to deliver a pulsatile flow (130 pulses $/ \mathrm{min}$ ) of $0.9 \%$ saline solution from a reservoir. The flow rate was set to $1800 \mathrm{~mL} / \mathrm{min}$; the systolic pressures were measured using a system for intra-arterial blood pressure monitoring (TruWave; EdwardsLifesciences, Irvine, Calif) and found to be $100 \mathrm{~mm} \mathrm{Hg}$. A polyethylene mesh filter with a 53- $\mu \mathrm{m}$ width (Nytal; Sefar, Heiden, Switzerland) was placed at the inflow side of the reservoir to capture the particles that passed through the detachable filter.

\section{Simulation of Thromboembolism}

Polystyrene-divinylbenzene microspheres (EZ-TRAC; IMT Laboratories, Irvine, Calif) with a diameter of 100 and $200 \mu \mathrm{m}$ were used. The microspheres were preserved in $0.9 \%$ saline, and the numbers of the 100 - and $200-\mu \mathrm{m}$ microspheres were 2000 and 250 , respectively, in $0.1 \mathrm{~mL}$ of solution. The microspheres, suspended in $10 \mathrm{~mL}$ of $0.9 \%$ saline, were inserted into the filter from a $6 \mathrm{~F}$ guiding catheter, set $5 \mathrm{~cm}$ proximal to the detachable filter.

After positioning the detachable filter, each microsphere was carefully injected into an aortic branch within 30 seconds using the $6 \mathrm{~F}$ guiding catheter. Next, residual microspheres in the guiding catheter were flushed out 3 times with $10 \mathrm{~mL}$ of $0.9 \%$ saline. After 5 minutes, the polyethylene mesh filter ( 53 $\mu \mathrm{m}$ in width) was removed, and the number of captured particles on the mesh was counted through a microscope. Particles captured by the $53-\mu \mathrm{m}$ mesh filter (after passing the detachable filter) were defined as distal emboli.

The capture efficacy was calculated as follows: capture efficacy $(\%)=$ number of particles trapped by the filter (total particles - particles captured by the $53-\mu \mathrm{m}$ mesh filter - particles left in syringe)/total particles $\times 100$.

Thus, the greatest possible capture efficacy was $100 \%$. Ten trials were conducted for each microsphere size.

\section{Implantation of Detachable Filter in Canine Model}

Five normal mongrel dogs, weighing $25-30 \mathrm{~kg}$ each, were used in our experiment. All dogs received humane care in compliance with the Principles

Frame ring

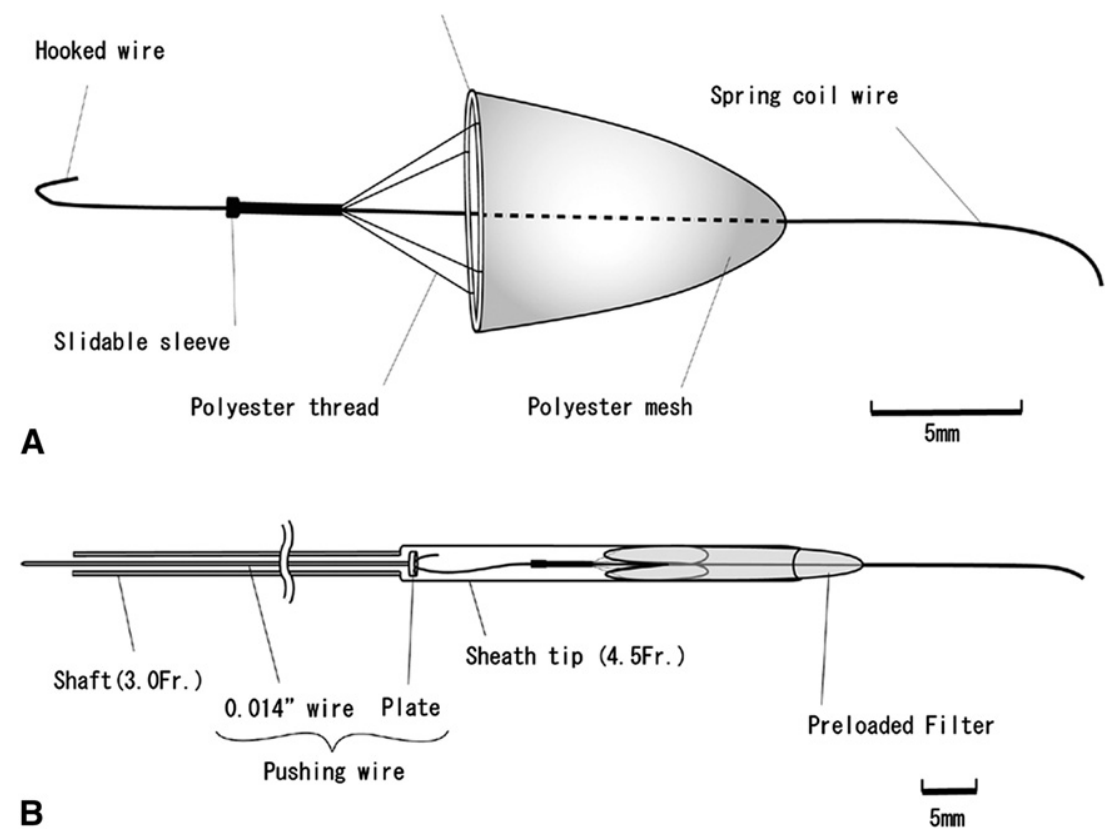

FIGURE 1. Schematic representation of the filter and the filter system. A, New detachable filter. B, Preloaded filter in the delivery sheath. 


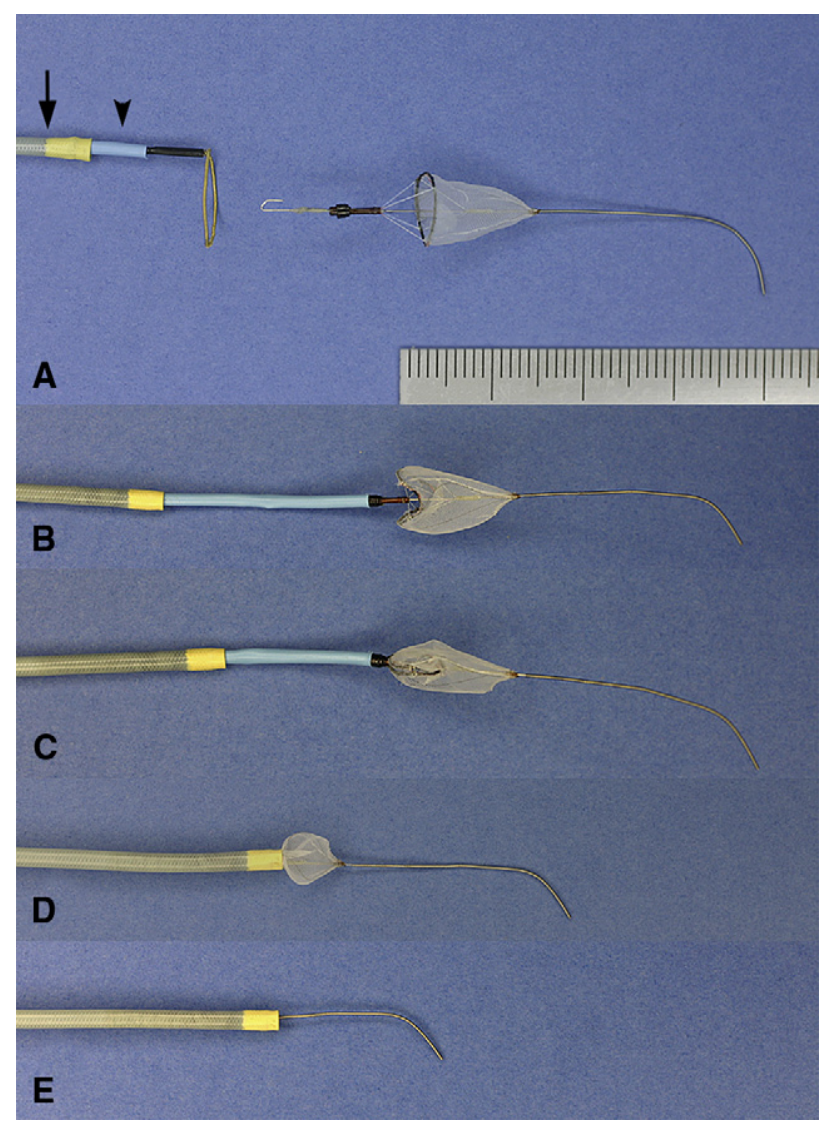

FIGURE 2. Various steps of the detachable filter retrieval. A, Approximation of a gooseneck snare to the filter. B and C, After capture of the hook of the filter, the slidable sleeve is moved distally by pushing the $4 \mathrm{~F}$ catheter, resulting in folding of the filter. D and $\mathrm{E}$, The folded filter device with $4 \mathrm{~F}$ catheter and gooseneck snare is retrieved into the $6 \mathrm{~F}$ guiding catheter. Arrow, $6 \mathrm{~F}$ guiding catheter; arrowhead, $4 \mathrm{~F}$ catheter. Scale, $1 \mathrm{~mm}$.

of Laboratory Animal Care formulated by the National Society for Medical Research and the Guide for the Care and Use of Laboratory Animals prepared by the National Academy of Sciences and published by the National Institutes of Health (Department of Health, Education, and Welfare, National Institutes of Health publication 85-23, revised 1985). The study was approved by the Kyoto University Research Animal Review Committee and conducted in accordance with the policies of Kyoto University.

Surgical preparation and anesthesia were performed as follows. The dogs were premedicated with intramuscular injections of xylazine (40 $\mathrm{mg}$ ) and atropine sulfate $(50 \mathrm{mg})$. Subsequently, an intravenous catheter was inserted into the right brachial vein, and propofol $(100 \mathrm{mg})$ was administered through the catheter. After inducing general anesthesia with pentobarbital, the dogs were maintained on ventilator support with $1 \%$ isoflurane in $100 \%$ oxygen. From the femoral artery, a $6 \mathrm{~F}$ sheath catheter was placed after systemic heparinization (100 U/kg). As described, the detachable filter was deployed into each targeted aortic branch under fluoroscopic guidance (OEC 9800 Plus; General Electric, Fairfield, Conn) using a contrast medium. Every 30 minutes, the contrast medium was injected through the guiding catheter, and its flow through the filter was investigated. At 5 hours after deployment, the filter was retrieved by a gooseneck snare catheter using the method previously shown.

\section{Histologic Examination}

After retrieval of the filter, the internal carotid artery of 1 canine was removed, perfusion fixed with $10 \%$ buffered formalin, and embedded in plastic. The sample was then divided into 3 segments: distal, central (containing the filter landing zone), and proximal. Each segment was stained with hematoxylin and eosin (Cole-Parmer Instrument, Vernon Hills, Ill).

\section{Statistical Analysis}

All values are expressed as the mean \pm standard deviation. The comparison of the 2 groups was analyzed using the Wilcoxon signed rank test. All statistical analyses were performed using the StatView software (SAS Institute, Cary, NC).

\section{RESULTS \\ In Vitro Studies}

No technical failures occurred during any of the procedures. The new detachable filter had a high capture efficacy, with no statistically significant difference between the $2 \mathrm{mi}-$ crosphere sizes tested. The mean efficacy was $99.2 \% \pm$ $0.6 \%$ for the $100-\mu \mathrm{m}$ microspheres and $99.4 \% \pm 0.5 \%$ for the $200-\mu \mathrm{m}$ microspheres $(P>.05)$.

\section{In Vivo Studies}

All new detachable filters were successfully deployed in all aortic branches of 5 mongrel dogs. The J-shaped spring coil wire attached to the filter accurately conducted the filter to the targeted branch even when the branch was tortuous. The activated clotting time was maintained at 150 to 250 seconds during the procedure. Angiography, performed every 30 minutes for 5 hours, revealed smooth blood flow through the filter without any signs of obstruction or thromboembolism in any of the 5 dogs (Figure 4). All filters were completely retrieved using the gooseneck snare catheter without aortic injury. The mean interval to achieve deployment was $9.0 \pm 3.3$ minutes (range, 3-14), and the mean duration for the retrieval was $9.6 \pm 6.2$ minutes (range, 3-20). As we gained technical experience, both these times progressively decreased.

\section{Histologic Findings}

Histologic analysis revealed complete preservation of the endothelium and intima, even where the filter ring had attached to the arterial wall. The media and adventitia were also preserved.

\section{DISCUSSION}

The main finding of the present study was that the detachable filter was successfully inserted and deployed in all major branches of the aorta. After 5 hours, the device was completely retrieved from the branches without any complications, such as extravasation, aortic dissection, or aortic rupture. The efficacy of the detachable filter was also demonstrated by the results from our in vitro studies. To the best of our knowledge, this is the first report of a detachable filter that can be applied to all major aortic branches with longterm durability.

Endovascular repair with stent grafts offers an attractive alternative to surgical repair for thoracic aortic aneurysms. ${ }^{1-3}$ 


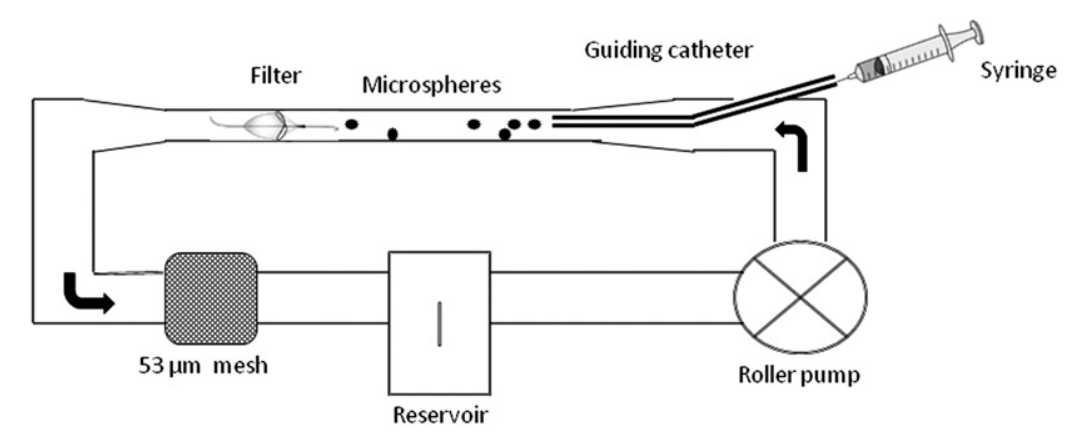

Capture efficacy $(\%)=\frac{\text { particles trapped by the filter }}{\text { total particles }} \times 100$

particles trapped by the filter $=$ total particles - particles in the $53-\mu m$ mesh filter - particles left in syringe

FIGURE 3. Schematic representation of experimental flow model.

In addition, percutaneous aortic valve replacement is a viable alternative to conventional open heart surgery in selected high-risk patients. ${ }^{4}$ However, distal thromboembolism has still been reported as a complication during these procedures. $^{5-8}$ Our efficient filter could contribute to reduce the risk of distal thromboembolism. Moreover, during endovascular surgery, such as aortic stent grafting or transcatheter aortic valve implantation, no wires and catheters are placed in the thoracic aorta when our detachable filters are deployed directly into the aortic branches. Thus, these detachable filters might not disrupt the main endovascular surgery.

Commercially available distal protection filters provide only short-term protection. This short lifespan might be due to the relatively low fraction of pore space in each filter; thus, thrombus captured by the filter might increase the resistance to blood flow. Our new filter is composed of polyester mesh and polyester yarn, $100 \mu \mathrm{m}$ and $27 \mu \mathrm{m}$ in diameter, respectively. The proportion of pores in the mesh filter is $62 \%$. This construction might help to maintain smooth blood flow and thus reduce the risk of distal ischemia.

The coating on the filter is significant in preventing obstruction of the filter itself. The application of an heparin coating alone on filter membranes might not be adequate for maintaining long-term filter performance. To prevent early occlusion, a new antithrombogenic coating system that possesses the inhibitory functions of both thrombin and platelet adhesion was introduced into the new detachable filter. This coating technology is based on the novel antithrombogenic polymer developed by Toray Industries. The polymer is able to reduce both the enzymatic activity of thrombin and platelet adhesion in the same molecule, and each function works independently on the material

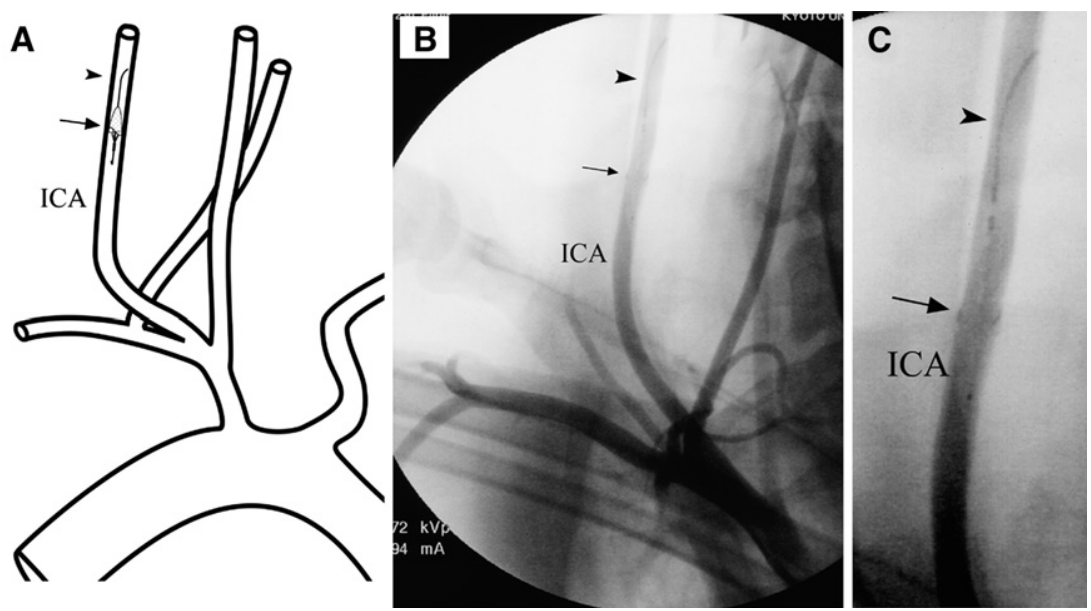

FIGURE 4. Angiographic images revealing the new filter in an internal carotid artery. A, Schematic and B, angiographic representations of filter deployment and $\mathrm{C}$, its partial magnification. Arrow, ring of the filter; arrowhead, spring coil; ICA, internal carotid artery. 
surface. An extremely thin layer of the coating was applied to the filter. This thin coating does not decrease the pore size, yet provides the benefit of preventing thrombus adhesion.

Another remarkable advantage of our detachable filter is that its ring can be folded inward during retrieval. This feature would help to reduce the scattering of thrombus that have attached to the edge of the filter. One of the shortcomings of our detachable filter is that it required a relatively long time to deploy and retrieve, although the time progressively decreased with increased technical experience.

Several studies have shown that distal protection devices are able to capture particles larger than the mesh pore size. ${ }^{12-14}$ Our study showed similar results, because our detachable filter was able to capture particles of both sizes with high efficacy.

The detachable filters were successfully deployed into each branch of the canine arteries and were retained in place for 5 hours before retrieval. This suggests that our novel filter device might be tolerated for all endovascular surgeries. Although our histologic analysis showed complete preservation of the endothelium and intima, the possible risks of iatrogenic injury and aortic dissection should be duly considered.

\section{Study Limitations}

The present study had several potential limitations. First, the anatomy of the aortic wall is not identical between humans and canines. The diameters of the canine arteries such as the carotid, celiac, and supramesenteric arteries, are as small as $6 \mathrm{~mm}$; the differences are greater when canine and human aortas are compared. The manipulation of the filter device in a human arteriosclerotic aorta could be much more complicated than that in a normal, undiseased aorta. However, the angles between the aorta and the branching arteries are convergent; therefore, the technical difficulties in deploying and retrieving the detachable filter could be assumed to be identical. The second limitation was the length of the shaft. The form of the polyester mesh was designed according to the length of the shaft. If the shaft were shorter, the volume of the mesh filter would be reduced. The present study was also limited to a single filter size. Filters of different shapes might produce different results; therefore, a more detailed examination is necessary to determine the feasibility of using detachable filters of different sizes.

In conclusion, we have developed a novel detachable filter, successfully deployed it for 5 hours in all major aortic branches, and then retrieved it without any complications. This novel filter could help prevent serious thromboembolism during all types of endovascular surgery. More experimental studies and device improvements are required before this technique will be ready for clinical use in humans.

\section{References}

1. Criado FJ, Abul-Khoudoud OR, Domer GS, McKendrick C, Zuzga M, Clark NS, et al. Endovascular repair of the thoracic aorta: lessons learned. Ann Thorac Surg. 2005;80:857-63.

2. Czerny M, Funovics M, Sodeck G, Dumfarth J, Schoder M, Juraszek A, et al Long-term results of thoracic endovascular aortic repair in atherosclerotic aneurysms involving the descending aorta. J Thorac Cardiovasc Surg. 2010;140: 179-90.

3. Kuratani T, Sawa Y. Current strategy of endovascular aortic repair for thoracic aortic aneurysms. Gen Thorac Cardiovasc Surg. 2010;58:393-8.

4. Webb JG, Pasupati S, Humphries K, Thompson C, Altwegg L, Moss R, et al. Percutaneous transarterial aortic valve replacement in selected high-risk patients with aortic stenosis. Circulation. 2007;116:755-63.

5. Inoue K, Hosokawa H, Iwase T, Sato M, Yoshida Y, Ueno K, et al. Aortic arch reconstruction by transluminally placed endovascular branched stent graft. Circulation. 1999;100:316-21.

6. Schoder M, Lammer J, Czerny M. Endovascular aortic arch repair: hopes and certainties. Eur J Vasc Endovasc Surg. 2009;38:255-61.

7. Zempo N, Sakano H, Ikenaga S, Harada M, Yamashita A, Morikage N, et al. Fatal diffuse atheromatous embolization following endovascular grafting for an abdominal aortic aneurysm: report of a case. Surg Today. 2001;31: 269-73.

8. Obitsu Y, Koizumi N, Iida Y, Iwahashi T, Saiki N, Watanabe Y, et al. Long-term results of second-stage thoracic endovascular aortic repair following total aortic arch replacement. Gen Thorac Cardiovasc Surg. 2010;58:501-5.

9. Schreiber TL, Strickman N, Davis T, Kumar V, Mishkel G, Foster M, et al. CASES-PMS Investigators. Carotid artery stenting with emboli protection surveillance study: outcomes at 1 year. J Am Coll Cardiol. 2010;56:49-57.

10. Massop D, Dave R, Metzger C, Bachinsky W, Solis M, Shah R, et al., SAPPHIRE Worldwide Investigators. Stenting and angioplasty with protection in patients at high-risk for endarterectomy: SAPPHIRE Worldwide Registry first 2,001 patients. Catheter Cardiovasc Interv. 2009;73:129-36.

11. Barbato JE, Dillavou E, Horowitz MB, Jovin TG, Kanal E, David S, et al. A randomized trial of carotid artery stenting with and without cerebral protection. J Vasc Surg. 2008;47:760-5.

12. Siewiorek GM, Wholey MH, Finol EA. In vitro performance assessment of distal protection filters: pulsatile flow conditions. J Endovasc Ther. 2009;16:735-43.

13. Finol EA, Siewiorek GM, Scotti CM, Wholey MH, Mholey MH. Wall apposition assessment and performance comparison of distal protection filters. $J$ Endovasc Ther. 2008;15:177-85.

14. Müller-Hülsbeck S, Stolzmann P, Liess C, Hedderich J, Paulsen F, Jahnke T, et al Vessel wall damage caused by cerebral protection devices: ex vivo evaluation in porcine carotid arteries. Radiology. 2005;235:454-60. 